\title{
Application of Soft Computing Technologies toward Assessment and Skills Development
}

\author{
Ignacio Aliaga1, Vicente Vera ${ }^{1}$, Cristina González Losada ${ }^{1}$, Álvaro Enrique \\ García ${ }^{1}$, Héctor Quintián², Emilio Corchado², Fanny Klett ${ }^{3}$, Laura García- \\ Hernández $^{4}$
}

\begin{abstract}
Schools and universities face multiple challenges when they target initiating or expanding undergraduate programs. Education has traditionally utilized a teacher-centered educational and assessment approach. Only few attempts exist to involve objective feedback and non-traditional assessment methods and technologies to improve the processes of teaching, learning, and education in general.

This paper addresses a novel objective multi-parameter assessment methodology based on Soft computing technology to discover the effect of students' groupings by exploiting the interrelationships between the grades the students received for their laboratory subjects and the grade they obtained in the university enrolment exam. The research results allow for exploring non-desirable discordant teaching and assessment practices for individuals or groups. In addition, the results obtained illustrate opportunities to focus on the individual student during the education process and determine adaptive teaching strategies based on the particular level of knowledge and experience. Toward these results statistical and Soft computing models implementing Unsupervised Neural and Exploratory Projection Techniques have been applied to carry on the objective assessment of the students' skills development during the entire higher education period.

Empirical verification of the proposed assessment model is performed in a real environment, where a case study is defined, and analysed. The real data set to validate the performance of the proposed approach has been collected at the School of Dentistry of the Complutense University of Madrid.
\end{abstract}

Keywords: Component, Unsupervised Neural and Exploratory Projection Techniques, Assessment of skills development, Higher education and training, Dental milling.

\footnotetext{
${ }^{1}$ University Complutense of Madrid, Odontology Faculty, Madrid, Spain Ialia01@estumail.ucm.es, \{vicentevera,aegarcia\}@odon.ucm.es

${ }^{2}$ University of Salamanca, Departamento de Informática y Automática Plaza de la Merced s/n, 37008, Salamanca, Spain \{hector.quintian, escorchado\}@usal.es

${ }^{3}$ Director, German Workforce ADL Partnership Laboratory, Germany fanny.klett.de@adlnet.gov

${ }^{4}$ Area of Project Engineering, University of Cordoba, Spain ir1gahel@uco.es
} 


\section{Introduction}

In the past, the term assessment was used to refer particularly to the process of determining the extent to which learners have mastered a subject. It has recently been extended in the academic context to cover all uses of evaluation. Assessment data are observations or facts that must be collected, organized, and analysed to become useful.

Assessment has many roles. On the one hand, it is critical for measuring the student's achievement within the learning process, i.e. students can understand to what extend learning objectives have been accomplished. On the other hand, it can also be used as a teaching tool to help students better understand concepts and enhance their skills. In addition, the instructor can realize what students are understanding as well as the concepts that need further explanation. Thus, assessment can be viewed as a powerful tool for quality management, on the individual and the organizational level. [1] Simultaneously, it appears as part of the skills development process. In the framework of skills development and career support, assessment needs a systematic approach to develop a range of transferable personal skills that are sought by every employer, and to track skills development and competency data for every student.

Schools and academia are particularly aware that applying sustainable assessment processes will support them in treating the new challenges that arise every year when trying to improve their educational programs and adapt to the needs of the market, industry and businesses. Innovative approaches in teaching and learning enable handling various complex considerations in engineering, medicine, dentistry and further curricula where various psychomotor, modelling, and complex problem solving skills have to be addressed in practical settings. The acquisition of such skills and abilities appears particularly important for the development of a wide variety of career paths within engineering, design and medicine and requires enormous willingness and individual training [1] leading to a specific psychomotor development, which is determined by the innate abilities and the learning results achieved during the training process [2,3]. The objective assessment of the psychomotor skills remains a challenge, and requires new models and approaches, particularly different from assessing learning in general. Moreover, the requirement for tracking and analysing assessment data in due course creates the demand for an effective technology based assessment.

Against this background, Soft computing and statistical models offer a sufficient environment to address multi-parameter assessment [4-8] of psychomotor skills due to the integrated beneficial opportunity for visualization of analytical and multidimensional data able to facilitate the interpretation of results, and thus, improve the effectiveness of decision-making processes [9]. It appears advantageous to apply Soft computing models to assessment, based also on results from social and collaborative learning theories. For example, knowing the level of psychomotor development of an individual in relation to other students in the same class, it is possible to identify individuals who experience larger difficulties. Once these difficulties are identified, the instructors can raise individual and collective reinforcement [10]. 
This paper presents a precise, logical, achievable, observable, and objectively measurable Soft computing model to analyse the skills development of students based on a comprehensive analysis of the psychomotor skills of an individual compared to the skills of classmates. This model enables the proper assessment of the skills development process in a practical setting by correlating variables such as the age of the students, previous experience, etc. [11].

The next sections refer to main aspects of the novel Soft computing model to facilitate and advance the assessment of the skills development process. Testing and verification of the approach is performed in a real case study involving dentistry students. The technology has been developed at the Complutense University of Madrid (Test Skills Practice).

\section{The General Soft computing Model}

Engineering, design and medicine curricula involve a series of practical experiences in the area of psychomotor skills development referring to an orderly and chained sequence, and following a series of learning objectives that help the instructors to organize them. Each of these practical experiences includes the criteria for assessing the achievement of the objectives.

Commonly, the learning objective is a statement that describes what a learner will be able to do as a result of learning and/or teaching. A learning objective should be precise, logical, achievable, observable and measurable, and it becomes an inseparable part of the assessment process that can involve various variables depending on the evaluation aims. For example, a comprehensive analysis of the individual abilities of a student compared with the classmates' ones and measuring the learning achievements after a period of time without having received more learning, is a challenging task. It is valuable also to analyse all the skills the student has developed during an entire undergraduate study toward identifying a relationship between the grade obtained in the university enrolment exam, the continuing psychomotor skills performance and the ease of learning. Variables such as the age of the students when starting a course and their previous experience in a similar field, is a factor enormously influencing the skills development process.

Exploratory Projection Pursuit (EPP) [12] is a statistical method for solving the difficult problem of identifying structure in high dimensional data. The method used here is based on the projection of the data onto a lower dimensional subspace in which we search for its structure with the naked eye. It is necessary to define an "index" that measures the interestingness of a projection. After that the data is transformed by maximizing the index in order to maximise interest according to that index. From a statistical point of view the most interesting directions are those which are as non-Gaussian as possible [13]. The Soft computing model takes advantage of a neuronal model of Exploratory Projection Pursuit (EPP), and Maximum-Likelihood Hebbian Learning (MLHL) $[12,14]$ to best match the criteria identified for assessing the psychomotor skills of students performing practical work.

To test and verify the research approach, a real case study was developed by focusing on dentistry students studying the subject Introduction to Dentistry in the 
undergraduate degree of Dentistry. The practical work refers to the performance of cavities, of shapes with different levels of difficulty, previously designed on methacrylate blocks by using two techniques (high speed -turbine- and low speed contra-). The challenging factor for the Soft computing model consists in the fact that a number of students have been accessed after completing Dental Hygienist or Dental Technician degrees. In light of the above, the variables mentioned before are particularly interesting to be explored during the entire undergraduate study because they may raise the hypothesis that the psychomotor skills of these students vary from the skills of the remaining undergraduates.

The next section presents the intelligent model applied for clustering students. It is based on the use of projection models.

\section{The Specific Soft Computing Model - An Intelligence System for Clustering Students}

This section introduces the main intelligent model applied in this research to perform clustering in the students' data set. It is used to provide a visual analysis of the internal structure of the data set.

A combination of projection types of techniques together with the use of scatter plot matrices constitute a very useful visualization tool to investigate the intrinsic structure of multidimensional datasets, allowing experts to study the relationship between different components, factors or projections, depending on the technique used.

Projection methods help projecting high-dimensional data points onto lower dimensions in order to identify "interesting" directions in terms of any specific index or projection. Such indexes or projections are, for example, based on the identification of directions that account for the largest variance of a dataset (such as Principal Component Analysis (PCA)[15]) or the identification of higher order statistics such as the skew or kurtosis index, as in the case of Exploratory Projection Pursuit (EPP) [16]. Having identified the interesting projections, the data is then projected onto a lower dimensional subspace plotted in two or three dimensions, which makes it possible to examine its structure with the naked eye. The remaining dimensions are discarded as they mainly relate to a very small percentage of the information or the dataset structure. In that way, the structure identified through a multivariable dataset may be visually analysed in an ease manner.

One of those models is the standard statistical EPP method [16], which provides a linear projection of a dataset, but it projects the data onto a set of basic vectors, which best reveal the interesting structure in data; interestingness is usually defined in terms of how far the distribution is from the Gaussian distribution.

The neural implementation of EPP, applied in this research for identifying the different students clusters, is the Maximum-Likelihood Hebbian Learning (MLHL) [14,17], which identifies interestingness by maximising the probability of the residuals under specific probability density functions that are non-Gaussian. 
Considering an N-dimensional input vector (x), and an M-dimensional output vector $(y)$, with $\mathrm{W}_{\mathrm{ij}}$ being the weight (linking input $\mathrm{j}$ to output $\mathrm{i}$ ), then MLHL can be expressed as:

1. Feed-forward step:

$y_{i}=\sum_{j=1}^{N} W_{i j} x_{j}, \forall i$

2. Feedback step:

$e_{j}=x_{j}-\sum_{i=1}^{N} W_{i j} y_{i}, \forall j$

3. Weight change:

$\Delta W_{i j}=\eta \cdot y_{i} \cdot \operatorname{sign}\left(e_{j}\right)\left|e_{j}\right|^{p-1}$ [13].

Where: $\eta$ is the learning rate and $p$ is a parameter related to the energy function

Then, by maximizing the likelihood of the residual with respect to the actual distribution, we are matching the learning rule to the probability density distribution of the residual. The power of the method comes from the choice of an appropriate function.

\section{The Real Case Study}

Significance of objective measurements has risen. Simultaneously, the prediction obtained by these measurements appears relevant to be exploited toward the assessment skills development. This fact also applies to dental students' [18].

The real case study focuses on facilitating the identification of divergent or non-desirable situations in the educational process. The particular aim of this study is to classify the psychomotor skills of first year students when creating methacrylate figures during this Dental Aptitude Test, which consists of carving ten methacrylate figures by using rotatory systems, and applying two different speeds (V1 and V2). A total of 20 figures are included. V1 (low speed) rotates at a speed of 10-60,000 revolutions per minute (rpm), while V2 (turbine or high speed) rotates at a speed of $250,000 \mathrm{rpm}$.

Seven of the figures made by the students can be easily created, while the remainders, which have several planes, involve a higher level of difficultly.

The tests conducted provide a comprehensive analysis of the students' performance with regard to their peer group and year of graduation, and allow for measuring and assessing the students' skills development during the course of an aca- 
demic year compared to other academic years, or to other students in the same academic year.

\subsection{Training Scenario Description}

Every student works on a methacrylate sheet at two different speeds, low speed and high speed. The low speed (10.000-60.000 rpm) is to be used to carve the first set of ten figures (see Fig 1). After completing this part of the practical work, students start carving the second part, which is basically a second round of the same figures, but this time using the high speed (150.000-250.000 rpm). The second instrument involves a higher level of difficulty as the bur is spinning faster and better psychomotor skills are the pre-requisite for effectively completing this task.

Both parts of the practical work have to be completed during 90 minutes and the results have to be submitted to the supervisors.

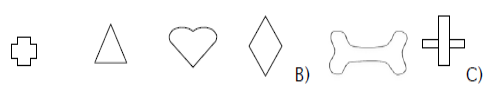

Fig. 1. Figures to be carved by the students.

The individual training involves the following steps:

- Properly assemble the low speed and turbine and test its operation. Recognize and properly mount the milling cutters.

- Create figures in a methacrylate block (see Fig 2). In advance, the students have to draw the outline of what to carve with a pen and a ruler.

- At the end of the training period the students have to submit their methacrylate block with all the figures carved on it (see Fig 2).Explanation:

o Figures should have an approximate size of $1 \mathrm{~cm}$ (in the length) and a depth of about 3-4 mm, except figures A, B and C (see Fig 1).

o The cavity design must be clear and free of defects, and the walls must be smooth and perpendicular to the surface of the methacrylate sheet. The floor must be parallel to the surface and as smooth as possible.

o Cavities are initially carved with a low speed, and subsequently with a turbine, related to the psychomotor skills acquired.

o Figures A, B and C (see Fig 1) have to be created in the end because of their level of difficulty concerning two planes.

o Students can repeat carving the figures as often as they like, so they can use all the figures but only one side of the methacrylate block.

o If the milling cutter is dulled with resin, it can be cleaned by spinning briefly and gently against a small block of wood or another milling cutter cleaner. 

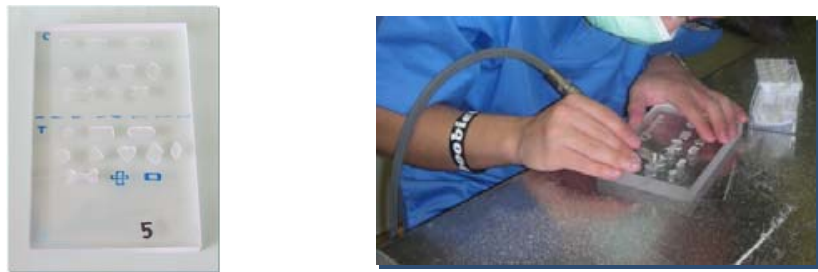

Fig. 2. Real methacrylate sheet with figures carved on it.

\subsection{Empirical Training Scenario Evaluation}

The real case scenario is empirically evaluated based on a survey of 79 first year dental students (24 students were eliminated from the initial 103 students, because they did not participate in the practical work). The information analysed for each student is based on 88 variables. The first most important eight variables concern:

- Age of the student (integer value).

- Sex of the student (integer value).

- Mark obtained by the student in the university enrolment exam. (Decimal value between 0 and 14).

- Previous experience gained by the student. The students may have had professional experience as a nurse, dental technician, hygienist, dental technician and hygienist, or lack of previous work experience.

- Mark obtained by the student in the theoretical exam of the subject (Decimal value between 0 and 10).

- Mark obtained by the students in the practical part of the subject (Decimal value between 0 and 10).

- Group class of the student (integer value between 1 and 4).

- Number of figures carved by the student (integer value between 0 and 20).

The following 80 variables (20 figures with four variables each) are the evaluations of the different parts of the figures (graded between 0 and 5). The way to interpret these variables is as follows: ' $x$ ' indicates the figure number and can range from 1 to 10 , ' $y$ ' indicates the speed used to carve the figure by using Low Speed (1) or High speed (2), and ' $z$ ' indicates the evaluator who examines the test ( 1 or 2):

- $\quad$ Fx_Vy_Ez_WALL: evaluate the quality of the walls of the figure created by the student.

- Fx_Vy_Ez_DEPTH: evaluate the quality of the depth of the figure created by student.

- Fx_Vy_Ez_EDGES: evaluate the quality of the edges of the figure created by student.

- Fx_Vy_Ez_FLOOR: evaluate the plain and irregularities presented on the floor of the figure created by the student. 
The data are collected in a document that represents the dataset to be evaluated and analysed.

The dataset, along with the corresponding labels, are recorded in a Comma Separated Value (CSV) format text file serving as input data in the software that applies the above described reduction treatment and generates the graphic representations.

\section{Results and Discussion}

Following the analysing procedure, PCA identified two clearly separated clusters called G1 and G2 (see Fig 3).

- $\mathbf{G}_{1}$ : This cluster represents students with high marks in the university enrolment exam, good or very good marks in the theoretical and practical matters of the subject, and a large number of figures carved. This cluster is composed by two sub-clusters: $\mathrm{C} 1$ and $\mathrm{C} 2$, as explained below.

- $\mathbf{G}_{2}$ : This cluster represents students with decent marks in theoretical and practical matters of the subject, and a small number of figures carved. This cluster is composed of two sub-clusters: C3 and C4, as explained below.

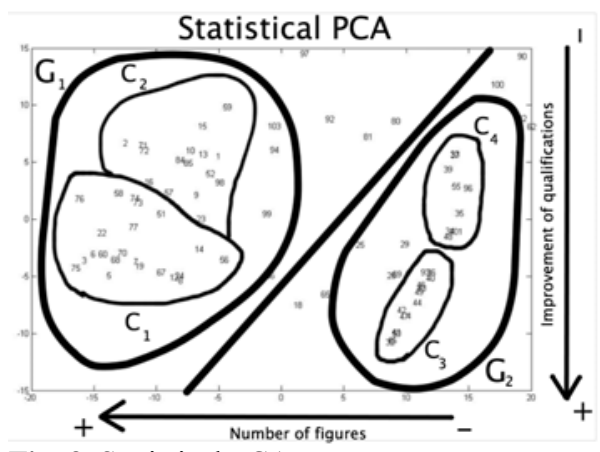

Fig. 3. Statistical PCA.

The sub-clusters refer to the following:

- $\quad \mathbf{C}_{1}$ represents young students (see Table 1) characterized with high marks in the university enrolment exam, without previous professional experience, and good marks in both, theory and practice.

- $\quad \mathbf{C}_{2}$ represents young students (see Table 1) with no previous professional experience, with good marks in the theoretical part of the subject and av- 
erage marks in the practical part. These students have carved many figures, but less than students belonging to the earlier cluster $(\mathrm{C} 1)$.

- $\quad \mathbf{C}_{3}$ represents students (see Table 1) with decent marks, both in the theoretical and practical parts of the subject, and have succeeded in carving an average number of figures (about half), mostly with low speed. Most of these students belong to the group number G2.

- $\quad \mathbf{C}_{4}$ represents students (see Table 1) that have been able to successfully carve 7 figures. Their marks in theory and practice are varied.

Table 1 Cluster Classification (PCA).

\begin{tabular}{ll}
\hline Clusters & Students belonging to each cluster \\
\hline C1 & $3,5,6,7,8,12,19,22,24,51,58,60,67,68,70,73,74,75,76,77$ \\
C2 & $1,2,9,10,13,15,16,23,52,57,59,71,72,84,85,98$ \\
C3 & $4,32,36,40,41,42,43,44,45,46,47,49,50,93$ \\
C4 & $33,34,35,37,39,48,55,96,101$ \\
\hline
\end{tabular}

After analysing the data using PCA, MLHL is then applied to find possible improvements in the classification of the samples (students).

Once the dataset test is analysed using MLHL (see Fig 4), the best result can be obtained by applying the following parameters: 3 neurons in the output layer $(\mathrm{m})$, 100000 iterations, 0.006 as learning rate, and 1.1 as $p$ (see equation 1,2 and 3).

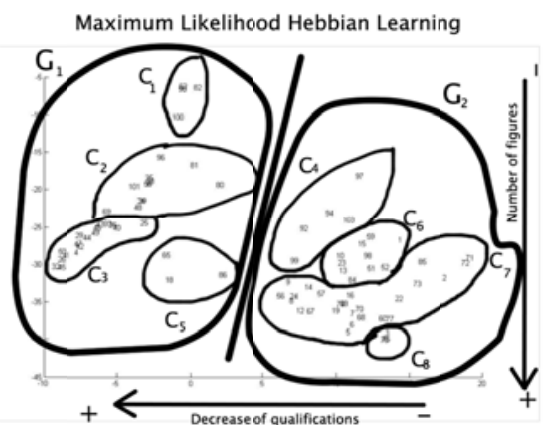

Fig. 4. MLHL Analysis with $\mathrm{m}=3$, iters $=100000$, lrate $=0.006$ and $\mathrm{p}=1.1$.

Like in PCA, two clusters $G_{1}$ and $G_{2}$ can be identified. In this case, the first cluster $\left(\mathrm{G}_{1}\right)$ is composed of four sub-clusters $\mathrm{C}_{1}, \mathrm{C}_{2}, \mathrm{C}_{3}$, and $\mathrm{C}_{5}$, and characterized by an average mark of less than 1.8 (marks go from 0 to 5 ) for the figures performed at high speed (V2). The second cluster $\left(\mathrm{G}_{2}\right)$ is composed of four sub- 
clusters $\left(\mathrm{C}_{4}, \mathrm{C}_{6}, \mathrm{C}_{7}\right.$ and $\left.\mathrm{C}_{8}\right)$ and characterized by an average mark greater than 1.8 for the figures carved at high speed (V2).

In order to facilitate the understanding of the results, the following equivalences are adopted from this point forward:

- Overall average: average of marks for figures created by a student.

- $\quad$ Average V1: average of marks for figures carved by a student using low speed.

- $\quad$ Average V2: average of marks for the figures created by a student using turbine (high speed).

The clusters that describe the data classification are the following:

- $\quad \mathbf{C}_{1}$ represents students (see Table 2) whose overall average is between 0.0 and 0.5 . This cluster is also characterized by an average V1 between 0 and 0.95 and an average V2 of mostly 0 .

- $\quad \mathbf{C}_{2}$ represents students (see Table 2) who have been able to successfully perform seven figures and whose overall average falls between 0.8 and 1.5. This cluster is also characterized by an average V1 between 1.7 and 2.95 and an average $\mathrm{V} 2$ of mostly 0.

- $\quad \mathbf{C}_{3}$ represents students (see Table 2) who have an overall average between 1.5 and 2.1. Students in this cluster are characterized by having made an average number of figures (between 8 and 13), most of which are carved with low speed (V1). This cluster is also characterized by an average V1 between 3 and 4.15 and an average V2 of mostly 0.

- $\quad \mathbf{C}_{4}$ represents students (see Table 2) who are similar to those belonging to the previous cluster, as the overall average of students falls within the same range, and the average V1 is a subinterval of students in cluster $\mathrm{C}_{2}$, namely between 1.64 and 2.48 . However, they differ in the average V2, because it falls within a completely different range (between 1.88 and 2).

- $\quad \mathbf{C}_{5}$ represents students (see Table 2) with an overall average similar to those in clusters $C_{3}$ and $C_{4}$ and who have created 13 or 14 figures. The overall V1 is a sub-interval of the overall V1 of $\mathrm{C}_{3}$ students, but the overall V2 is between 0.9 and 1.7.

- $\quad \mathbf{C}_{6}$ represents students (see Table 2) with an overall average between 2.1 and 2.8 and who have created 14-20 figures. Although this range is included in the interval of the previous cluster, they are not the same because the overall V1 is between 2.4 and 3.5 and the overall V2 is between 2.4 and 3.15 . 
- $\quad \mathbf{C}_{7}$ represents students (see Table 2) with an overall average between 2.8 and 3.75. The average V1 is between 3.1 and 3.7 and the average V2 is between 2.05 and 3.9. This is the largest cluster detected.

- $\quad \mathbf{C}_{\mathbf{8}}$ represents students (see Table 2) with the best overall average (between 3.9 and 4.1). Likewise, the average for both V1 and V2 is greater than 3.9. These students have been able to perform (almost) all the figures.

The following table shows which students belong to each cluster:

Table 2 Cluster Classification (MLHL).

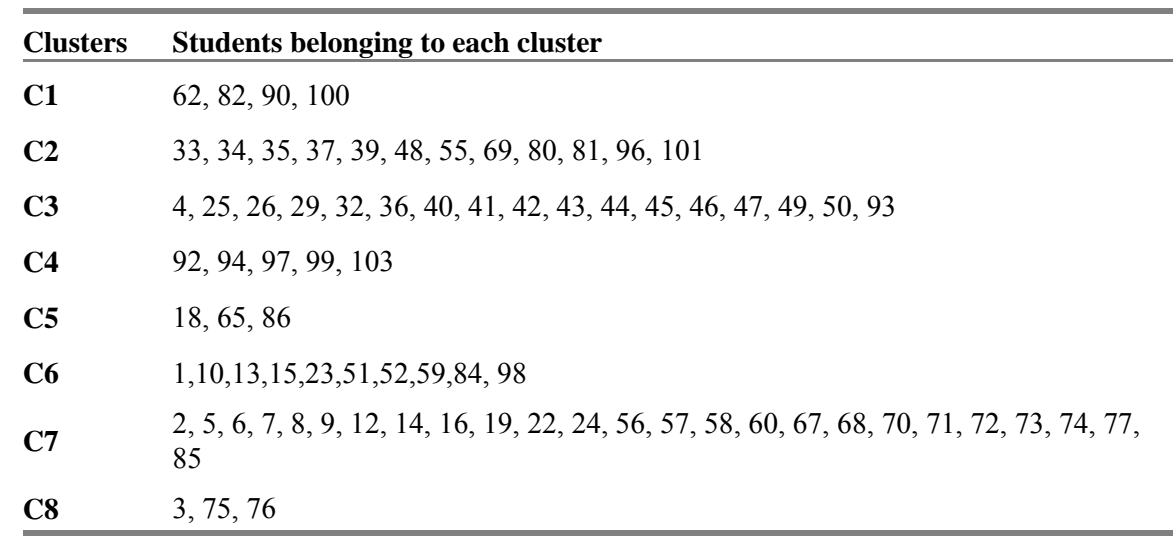

\section{Conclusions}

The analysis based on the use of PCA provides a valid initial classification of the real dataset, but it can significantly be improved by using MLHL.

The methodology applied provides promising results toward the comprehensive assessment of psychomotor skills development in career paths within engineering, design and medicine.

The novel model based on Soft computing technology has been verified in a real case study involving dentistry students. The variables analysed in this study can advantageously serve the teaching process by offering adaptive measures toward identifying those students most likely to pass the course, or who experience larger difficulties, respectively. According to the grade obtained in the university entrance exam and the number of figures carved, it's possible to predict clinical success the students' achievements in terms of reaching the highest mark in the Test Skills Practice. 
The research results make it possible to conclude that there is no relationship between recent achievements and previous professional experience, and this represents a helpful indication for the arrangement of future educational settings. Furthermore, this research shows that the quality of the figures carved is the better, the better the students have been trained in the preclinical period, i.e. the greater number of figures have been carved. Therefore, the practice appears a sustainable way to achieve clinical success.

Future work will focus on the extension and application of this objective assessment methodology based on Soft computing models in further educational fields and knowledge domains, such as civil, automotive, aerospace and mechanical engineering, and design and medicine to significantly improve the recent predominantly subjective practices of assessing skills development.

Acknowledgment. This research is partially supported through projects of the Spanish Ministry of Economy and Competitiveness [ref: TIN2010-21272-C02-01] (funded by the European Regional Development Fund). This work was also supported in the framework of the IT4 Innovations Centre of Excellence project, reg. no. CZ.1.05/1.1.00/02.0070 by operational programme 'Research and Development for Innovations' funded by the Structural Funds of the European Union and state budget of the Czech Republic, EU.

\section{References}

1. Klett, F.: The Design of a Sustainable Competency-Based Human Resources Management: A Holistic Approach. Special Issue of the Knowledge Management \& E-Learning. An International Journal, 3(2) (2010).

2. Grantcharov, T.P., Funch-Jensen, P.: Can everyone achieve proficiency with the laparoscopic technique? Learning curve patterns in technical skills acquisition. American Journal of Surgery, 197 (4), pp. 447-449 (2009).

3. Salgado, J., Grantcharov, T.P., Papasavas, P.K., Gagne, D.J., Caushaj, P.F.: Technical skills assessment as part of the selection process for a fellowship in minimally invasive surgery. Surg Endosc 23, pp. 641-644 (2009)

4. Polyzois, I., Claffey, I., McDonald, A., Hussey, D., Quinn, F: Can Evaluation of a Dental Procedure at the Outset of Learning Predict Later Per-formance at the Preclinical Level? A Pilot Study. European Journal of Dental Education. Blackwell Publishing Ltd, 15 (2), pp. 104-109 (2011)

5. Fiori, S.: Visualization of Riemannian-manifold-valued elements by multidimensional scaling. Neurocomputing. 74, pp. 983-992 (2011)

6. González-Navarro, F.F., Belanche-Muñoz, L.A., Romero, E., Vellido, A., Julià-Sapé, M., Arús, C.: Feature and model selection with discriminatory visualization for diag-nostic classification of brain tumors. Neurocomputing. 73, pp. 622-632 (2010)

7. Corchado, E., Herrero, Á.: Neural visualization of network traffic data for intrusion detection. Applied Soft Computing. 11, pp. 2042-2056 (2011)

8. Corchado, E., Sedano, J., Curiel, L., Villar, J.R.: Optimizing the operating conditions in a high precision industrial process using soft computing techniques. Expert Systems 29(3), pp. 276-299 (2012) 
9. Corchado, E., Perez, J.: A three-step unsupervised neural model for visualizing high complex dimensional spectroscopic data sets. Pattern Analysis \& Applications, 14, pp. 207-218 (2011).

10. Lee, T.Y., Radcliffe, D.F.: Innate design abilities of first year engineering and industrial design students. Design Studies, 11 (2), pp. 96-106 (1990)

11. Pitigoi-Aron, G., King, P.A., Chambers, D.W.: Predictors of Academic Performance for Applicants to An International Dental Studies Program in the United States. Journal of Dental Education, 75 (12), pp. 1577-82 (2011)

12. Friedman, J.H., Tukey, J.W.: A Projection Pursuit Algorithm for Exploratory Data Analysis. Computers, IEEE Transactions on. C-23, pp. 881-890 (1974)

13. Diaconis, P., Freedman, D.: Asymptotics of Graphical Projections. The Annals of Statistics, 12 (3), pp. 793-815 (1984)

14. Corchado, E., Pellicer, M.A., Borrajo, M.L.: A maximum likelihood Hebbian learning-based method to an agent-based architecture. International Journal of Computer Mathematics, 86, pp. 1760-1768 (2009)

15. Dorothy McComb, B.D.S.: Class I and Class II silver amalgam and resin composite posterior restorations: teaching approaches in Canadian faculties of dentistry. J Can Dent Assoc. 71 (6), 405-6 (2005).

16. Hotelling, H.: Analysis of a complex of statistical variables into principal components. Journal of Educational Psychology, 24 (6), pp. 417-441 (1933)

17. Corchado, E., MacDonald, D., Fyfe, C.: Maximum and Minimum Likelihood Hebbian Learning for Exploratory Projection Pursuit. Data Mining and Knowledge Discovery, 8, pp. 203-225 (2004)

18. Behar-Horenstein, L.S., Mitchell, G.S., Dolan, T.A.: A Case Study Examining Class-room Instructional Practices at a U.S. Dental School. J Dent Educ. 69, pp. 639-648 (2005) 